\title{
ANÁLISIS ESTRUCTURAL DE FALLAS GEOLÓGICAS Y POTENCIALES DESLIZAMIENTOS EN ANCONCITO, PROVINCIA DE SANTA ELENA
}

\author{
Kervin CHUNGA ${ }^{(1)}$ \\ Centro de Investigaciones de Geociencias CIGEO \\ Facultad de Ciencias de la Ingeniería \\ Universidad Estatal Península de Santa Elena UPSE \\ Campus La Libertad, vía principal Santa Elena - La Libertad
}

\begin{abstract}
Resumen
El sector costero de Anconcito, localizado al suroeste de la provincia de Santa Elena, es afectado por fenómenos geológicosinducidos por la dinámica costera de la zona de estudio: (1) socavamiento de los acantilados causado por la dinámica de la erosión marina y posterior derrumbe de estratos rocosos, y (2) deslizamientos de estratos rocosos a través de planos lístricos de fallas geológicas de tipo normal con componentes de desplazamientos laterales dextrales. El análisis estructural permitió definir el ambiente tectónico dominante con movimientos transtensivos asociados a esfuerzos de tracción.

Deslizamientos y desprendimientos de estratos rocosos acontecieron el 12 de Agosto de 2010 y el 7 de Abril de 2011, afectando directamente el sitio urbano "2 de febrero" y destruyendo redes eléctricas, camino vecinal y viviendas.

Análisis de morfometría del terreno permitió estimar 306.305 m3de material de terreno que puede deslizarse a través de 3 planos lístricos de fallas de tipo normal con efecto local de daño en un área de 14.585 m2.

Los factores que pueden incrementar la tasa de velocidad de deslizamiento de masas de tierra pueden ser: (1) altasprecipitaciones durante el fenómeno del Niño, y (2) la recurrencia de terremotos locales con magnitud moderada $\mathrm{Mw} \approx 5$ o el arribo de amplificaciones de ondas sísmicas desde terremotos lejanos con magnitudes mayores de $\mathrm{Mw}>7$. En conclusión, el sector de Anconcito debe ser motivo de análisis en detalle que permita establecer técnicas adecuadas para la recuperación de zona de playa y construcción de espigones que permitirán disminuir la alta energía de las ondas marinas.
\end{abstract}

Palabras Claves:Deslizamiento, falla normal, Anconcito, Santa Elena.

\begin{abstract}
The Anconcito coastal sector, located insouthwestern Santa Elena province, is affected by short duration geologic phenomena induced by the coastal dynamics in the area: (1) undermining of coastal cliffs, block toppling and rockfall which occur in the Anconcito sitemainly caused by the dynamics of the marine erosion, y (2) landslides of sandstone and siltstone strata are displacement through listric normal faults with right-lateral strike components. This structural analysis allow to define the dominant tectonic with transpressive displacement associated to extensional or normal strain.

Landslide and block toppling is what happened on August 12, 2010 and April 7, 2011, directly affecting the "2 de Febrero" site and destroying electric nets, road and housing.

The landslide volume could be roughly ca. $306.305 \mathrm{~m} 3$, this value is calculated by structural graphic based morphometric analysis and geological interpretation of digital terrain.

Another factors that may increase the rate of sliding of landmasses is rainfall associated with El Niño and,local recurrence of earthquakes with moderate magnitude $\mathrm{Mw} \approx 5$ amplifications or the arrival of seismic waves from distant earthquakes with magnitudes greater than $\mathrm{Mw}>7$.

In conclusion, Anconcito sector and chiefly in the "2 de Febrero" site should be subject to study in detail in order to establish proper techniques for the beach area recovery and construction of breakwaters that will reduce swells energy
\end{abstract}

Keywords: landslide, listric normal fault, Anconcito, Santa Elena 


\section{Introducción}

La Facultad de Ciencias de la Ingeniería de la Universidad Estatal Península de Santa Elena (UPSE) inició el estudio de vulnerabilidad costera en el sector urbano "2 de Febrero" en Anconcito, provincia de Santa Elena. La finalidad de este estudio surge del evento de deslizamiento del 12 de Agosto de 2010 y de la problemática de asentamientos urbanos en sitios no adecuado para la construcción y de alto riesgo geológico, rasgos como formación de grietas en el terreno y desprendimiento de estratos de rocas en los acantilados costeros de Anconcito son indicios de inestabilidad del talud.

Un primer reporte preliminar fue realizado el 27 de Agosto de 2010, en el que se estimó que: el área críticacomprende parte del sector poblado "2 de Febrero”. Desde un punto de vista de la geología estructural, esta área es delimitada por tresplanos de fallas geológicas de tipo normal, donde el área de influencia directa alcanza los14.585 m2; y el volumen de material que puede desplazarse a través de losplanos de fallas alcanzarían los 306.305 m3.

Modelos digitales del terreno evidencian planos de deslizamientos desde la base del acantilado hasta la vía pavimentada del Barrio "2 de Febrero".

Confirmando nuestro primer análisis geológico, un segundo evento aconteció a las 06h50 del 07.04.2011, el deslizamiento de estratos rocosos cambió bruscamente la morfología del terreno y permitió el colapso de viviendas e instalaciones de alumbrado eléctrico.

Este estudio no describe la cantidad de personas afectadas y re-ubicadas por parte de las entidades estatales pertinentes, sin embargo hace referencia a las familias que aún se encuentran asentadas en el área crítica de nivel alto de riesgo.

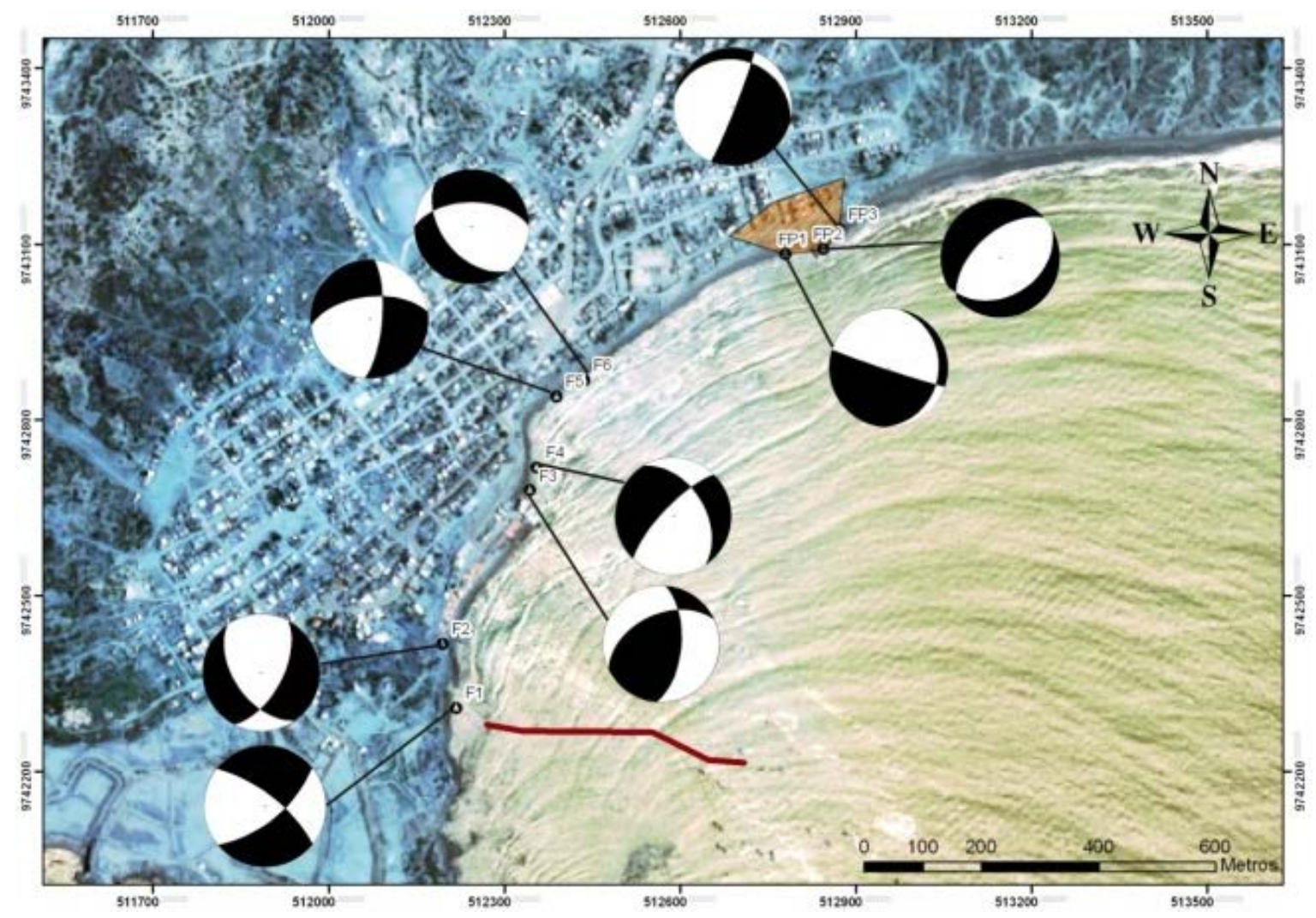

Figura 1. Imagen satelital de Anconcito analizada en plataforma GIS. Localización de estaciones de muestreos y representación de mecanismos focales para cada falla geológica individualizada durante la fase de geología de campo y exploración. La línea roja representa la distribución espacial de la escollera.

\subsection{Objetivo del trabajo}

Los principales objetivos de esta investigación son: (1) identificar el ambiente tectónico de la franja costera de Anconcito desde el sitio de la escollera hasta el sector periférico del sitio urbano " 2 de febrero”, (2) identificar estructuralmente la cinemática y la geometría de las fallas geológicas aflorantes en los acantilados, (3) identificar los fenómenos geológicos potencialmente activos con efectos de daños a infraestructuras urbanas construidas en la parte alta de los acantilados, y (4) calcular el área de influencia directa y volumen del material que se está desplazando a lo largo de planos de fallas geológicas. 


\subsection{Alcance de la investigación}

Como alcances fundamentales se detallan los siguientes logros: (1) Cálculo de volúmenes desplazados a lo largo de planos lístricosde fallas normales estimando a su vez la proyección de efecto en la superficie del terreno, delimitando así el área crítica, y (2) mapas de localización de los fenómenos geológicos representados por mecanismos focales desde el análisis estructural de planos de fallas geológicas.

\section{Método de estudio}

La metodología usada en este estudio incluye dos fases de investigación: (1) geología de campo y exploración, y (2) laboratorio y análisis geoestructural.
En la primera fase, la UPSE formó un equipo multidisciplinario de geólogos y geotécnicos, con uso de instrumentos de sistemas de posicionamiento global (GPS) y de Estación Total para delimitar geométricamente los fenómenos geológicos extremos, como aquellos identificados en el terreno: desprendimientos en bloques desde el borde del acantilado costero, y el desplazamiento de rocas a lo largo de planos de fallas geológicas.

La segunda fase comprende el procesamiento de datos compilados en la primera fase y la modelación digital del terreno distribuyendo espacialmente las estructuras geológicas delineadas en los acantilados costeros.

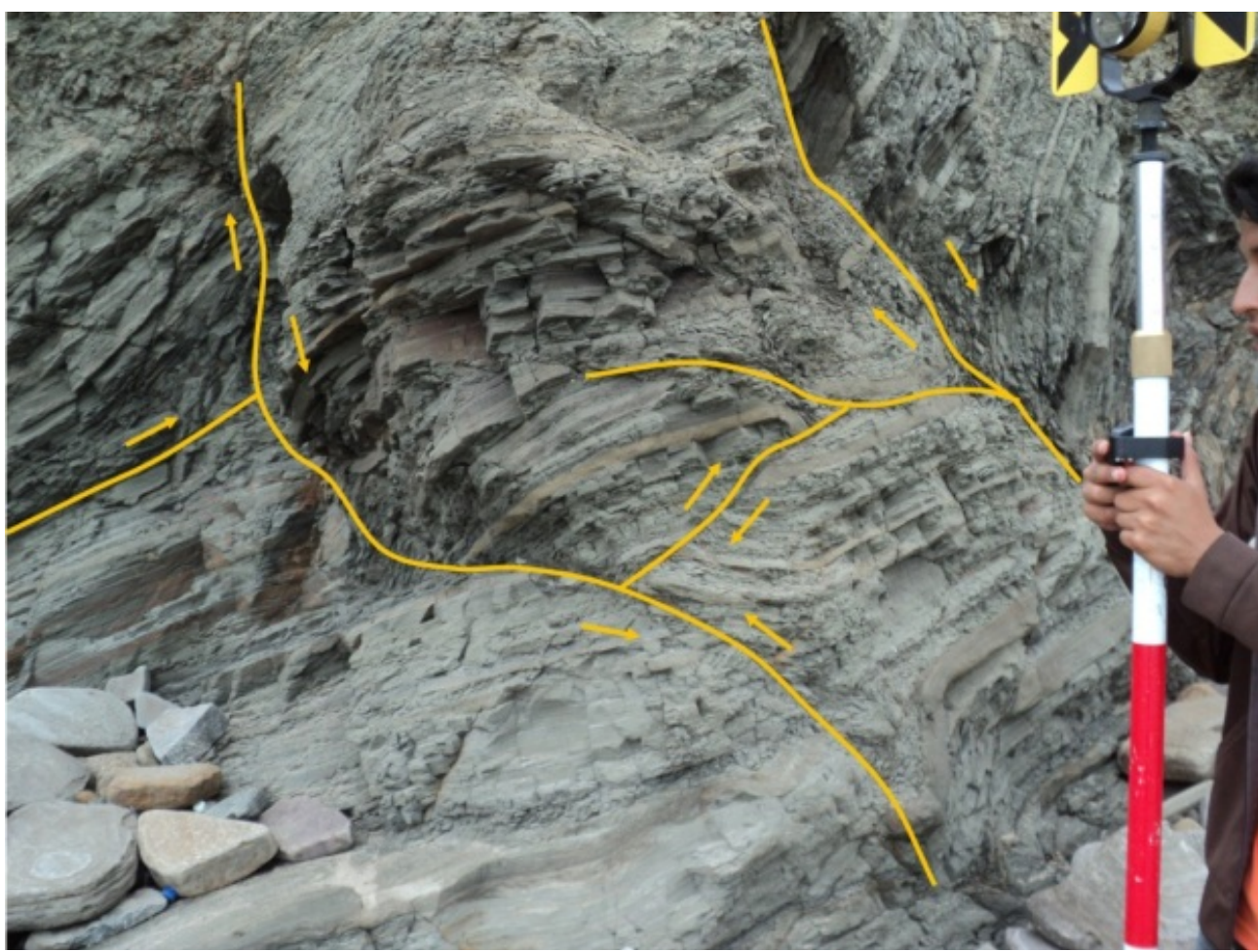

Figura 2. Microfallas asociadas a desplazamientos transpresivos de deformación sin-sedimentaria. La secuencia aflorante en el talud de Anconcito evidencia sistemas de fracturas con inestabilidad en zonas de pendientes.

\section{Geología local}

Durante la primera fase, se realizó un levantamiento geológico en la franja costera desde el sector de la escollera hasta los límites periféricos del sitio "2 de febrero" en Anconcito.

Un primer análisis, indica secuencias de estratos decimétricos de areniscas grauwáticas de clastossubangulares, intercalados por estratos laminados de arcillolitas y limolitas verdes grises afectadas por espejos de fricción observados en los afloramientos de los acantilados a lo largo de la estrecha zona de playa, además la plataforma litoral está compuesta litológicamente por este último tipo de roca. Nuñez et al. (1983) indica que esta secuencia sedimentaria corresponde al miembro "Arenisca" Punta Ancón de la Formación Seca (edad Eoceno Superior) del Grupo Ancón.

El Grupo Ancón agrupa tres formaciones geológicas: (1) ClayPebble Beds, (2) Socorro y Seca (Smith \& Williams, 1947), en Bristow \& Hoffstetter, 1977). Sin embargo, otra división geológica ha sido 
propuesta por Garner (1956) que incluye dos

$\begin{array}{ll}\text { Formación Seca: } & \begin{array}{l}\text { Miembro Arenisca Punta Ancón } \\ \text { Miembro Lutita Seca }\end{array} \\ \text { Formación Socorro: } & \begin{array}{l}\text { Miembro Socorro } \\ \text { Miembro ClayPebbleBed }\end{array}\end{array}$

En este estudio y para definir al Grupo Ancón, se ha adoptado las divisiones estratigráficas de Garner (1956), considerando que deslizamientos y fallas normales e inversas, debido a dos fases tectónicas de hundimientos y levantamientos, han producido inversiones estratigráficas en la cuenca Ancón.

En la localidad tipo Punta Ancón, aprox. 100 m de estratos suprayaciendo en contacto discordante sobre el precedente miembro Lutita Seca. Según Nuñez (1983), la litología es predominantemente areniscas grauwáticas con algunas capas arcillosas, conglomerados ligniticos (como los observados cerca del sitio escollera), capas calcáreas biohérmicas y lutitas interestratificadas. En este miembro (Punta Ancón) se incluye una arenisca blanca localmente brechosa de ceniza volcánica que aflorando en la zona de Punta Ancón; según evidencias de foraminíferos planctónicos y radiolarios que ocurren en las lutitas interestratificadas la edad es parecida a la del formaciones y cuatros miembros:

Miembro Seca, es decir Eoceno superior (ie. Bristow \& Hoffstetter, 1977).

Estructuralmente, en los afloramientos de los acantilados de Anconcito se evidencian estructuras plegadas "flame" y "convolucionadas" asociadas a microfallas inversas de deformación sin-sedimentaria por desplazamientos laterales transpresivos. No obstante, la tectónica actual indica otro mecanismo post-deformacional asociado a desplazamientos transtensivos caracterizados en el terreno por fallas normales con componentes destrales y sinestrales. Estas últimas son de interés técnico en el presente estudio, debido a los deslizamientos de masas de rocas a lo largo de estos planos de fallas con dirección hacia el S-SW están deformando y colapsando la superficie del terreno.

Ciertamente, nuestro análisis geotécnico permitió identificar dos principales fenómenos geológicos asociado a la tectónica actual: (1) desprendimientos en bloques en los bordes de los acantilados, y los más crítico (2) deslizamientos de masas rocosas a lo largo de tres principales (ie. FP1-FP2-FP3) planos de fallas geológicas de tipo normal. Este último fenómeno es considerado el de más alto riesgo geológico para la comunidad del sector " 2 de febrero" de Anconcito.

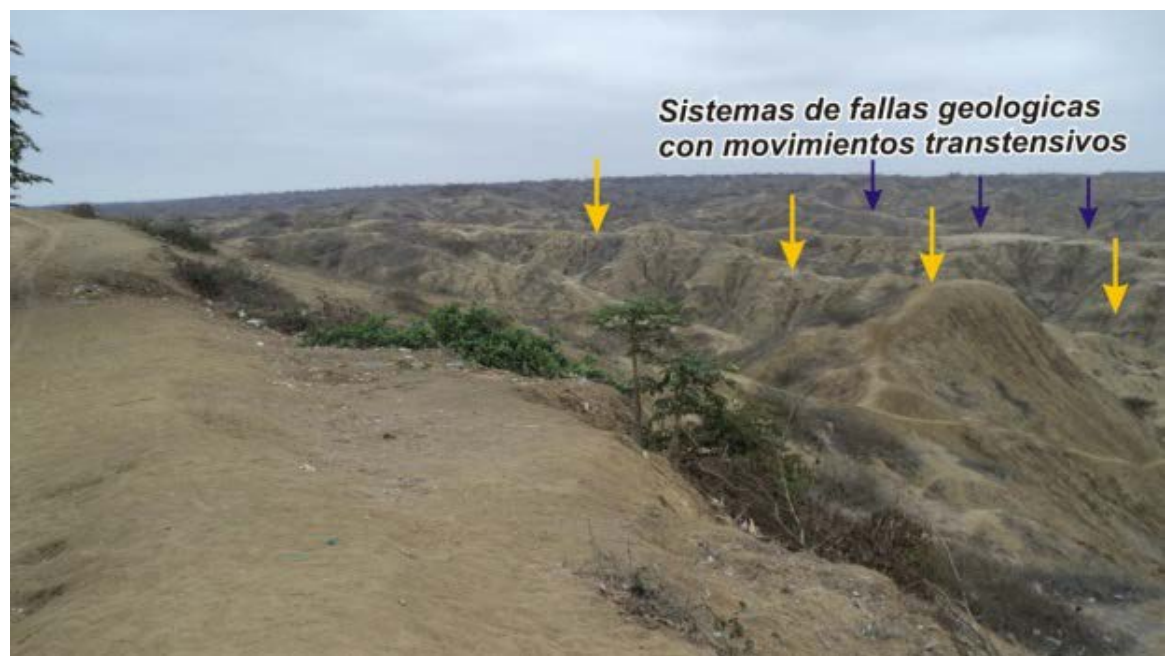

Figura 3. Sistemas de fallas geológicas capaces de deformar la superficie del terreno.

\section{Mecanismos focales $y$ campos de esfuerzos de fallas geológicas}

Como lo descrito en la sección 3, los mecanismos de deformación en las secuencias rocosas corresponden a dos episodios tectónicos de épocas diferentes, deduciendo en este estudio que las estructuras de pliegues y fallas inversas son asociadas a deformaciones sin-sedimentarias, ahora no activas y que pueden ser evidenciadas en los afloramientos de talud.

Con los primeros resultados obtenidos durante la primera fase de geología de campo, se permitió conocer la cinemática y geometría de nueve principales planos de fallas geológicas a lo largo del acantilado aflorante en la zona de playa, de las cuales todas tienen diferencias en sus tendencias estructurales y ocho de ellas son asociadas a movimientos transtensivos debido a los esfuerzos de tracción. Los datos estructurales son referenciados desde las estaciones F1-F2-F3-F4-F5-F6-FP1-FP2FP3 (ver, Figura 7).

Las estructuras de fallas que podrían ser consideradas activas y asociada a la tectónica actual son caracterizadas por movimientos transtensivos y representados gráficamente en la Figura 6 por mecanismos focales con dominio de campo de 
tracción. Estos mecanismos fueron generados con el software "Faultkin", donde los principales datos estructurales insertados son: rumbo, dirección de inclinación, ángulo de buzamiento y "Rake” dirección de fallas (indicados en la Figura 3). Este análisis corresponde a la fase de laboratorio y análisis estructural.

Estos resultados técnicos indican la inestabilidad del área de estudio y la posibilidad de que componentes externos como altas precipitaciones o atenuaciones de ondas sísmicas aceleren la velocidad de deslizamiento de las masas rocosas del sitio. También son considerados, otros factores como cercanas construcciones que emitan continuas vibraciones al terreno. De allí, la importancia de salvaguardar la integridad física de la comunidad afectada, y subsiguientemente la remoción de este material inestable, debido a que las rasgos evidenciados como grietas y planos de fallas (en abertura respecto a ambos bloques) no permiten una estabilización de talud.

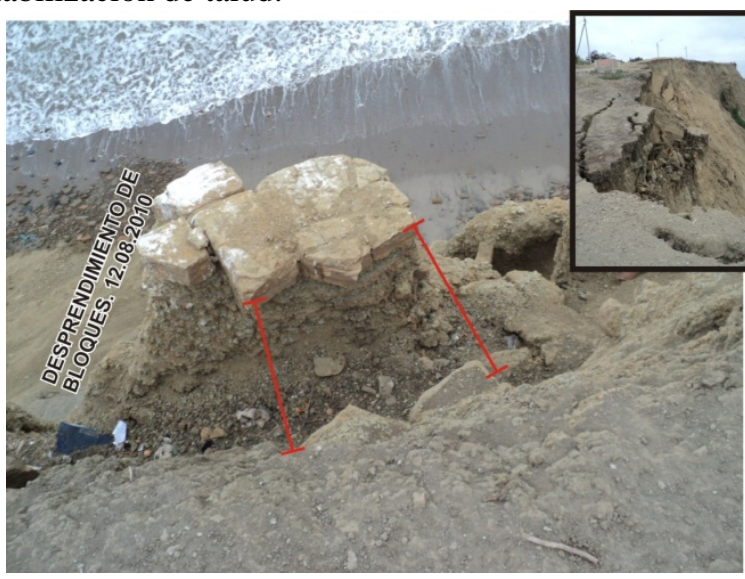

Figura 4. Desprendimiento en bloques de estratos rocosos de areniscas grauwáticas intercalada con laminaciones de arcillolita.

\section{Cálculo de volumen de material rocoso deslizándose a lo largo de planos de fallas geológicas}

Desde la obtención de datos de georeferenciación y proyectando los tres planos de fallas normales identificadas en este estudio, se estima que el área directamente afectada para la comunidad "2 de
Febrero" es de 14.585,96 m2 de material rocoso fragmentado.

El volumen total estimado que puede deslizarse con dirección SSE a través de los tres planos de fallas es de $306.305 \mathrm{~m} 3$. Desde un punto de vista de la morfología, este tipo de deslizamiento puede ser clasificado como rotacional, sin embargo es importante considerar la cinemática de las fallas geológicas presente en el área (de allí la importancia de conocer las diferentes direcciones de esfuerzos de tracción), ya que son los principales planos de deslizamientos que están deformando y colapsando el terreno.

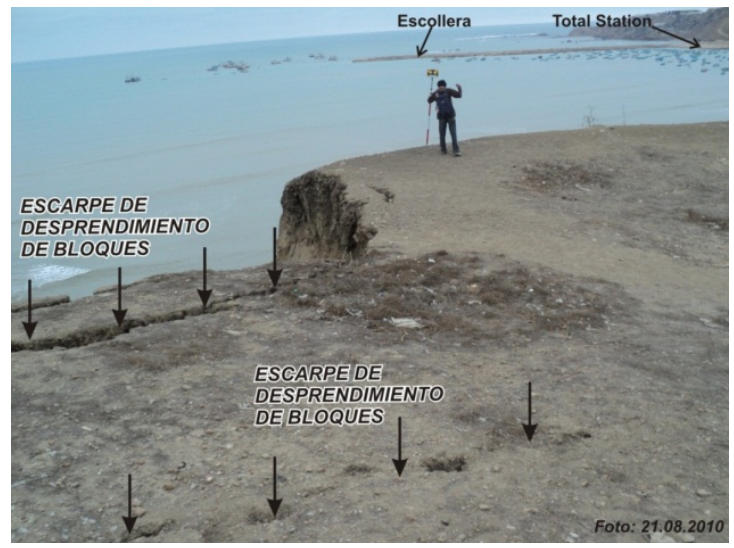

Figura 5. Evidencias de escarpes continuos de desprendimiento de bloques.

Estas estimaciones fueron realizadas desde datos de posicionamiento (GIS y Sistema de levantamiento altimétrico Estación Total), los cuales fueron distribuidos espacialmente a través de la aplicación del software ArcMap-GIS, de ese modo determinamos el área y volumen, esta información permite conocer la dimensión de deformación y a su vez los resultados puedan ser complementados para un estudio más a detalle, en la cual se podría definir técnicas ingenieriles adecuadas, para construcciones de escolleras (ie. adherentes, tipo $\mathrm{T}$, entre otras), las cuales tienen que ser posicionadas para recuperación de zona de playas y disminuir la dinámica de erosión marina en la base de los acantilados, donde se pierde continuamente resistencia de carga e incrementa la velocidad de desplazamiento de masas rocosas. 


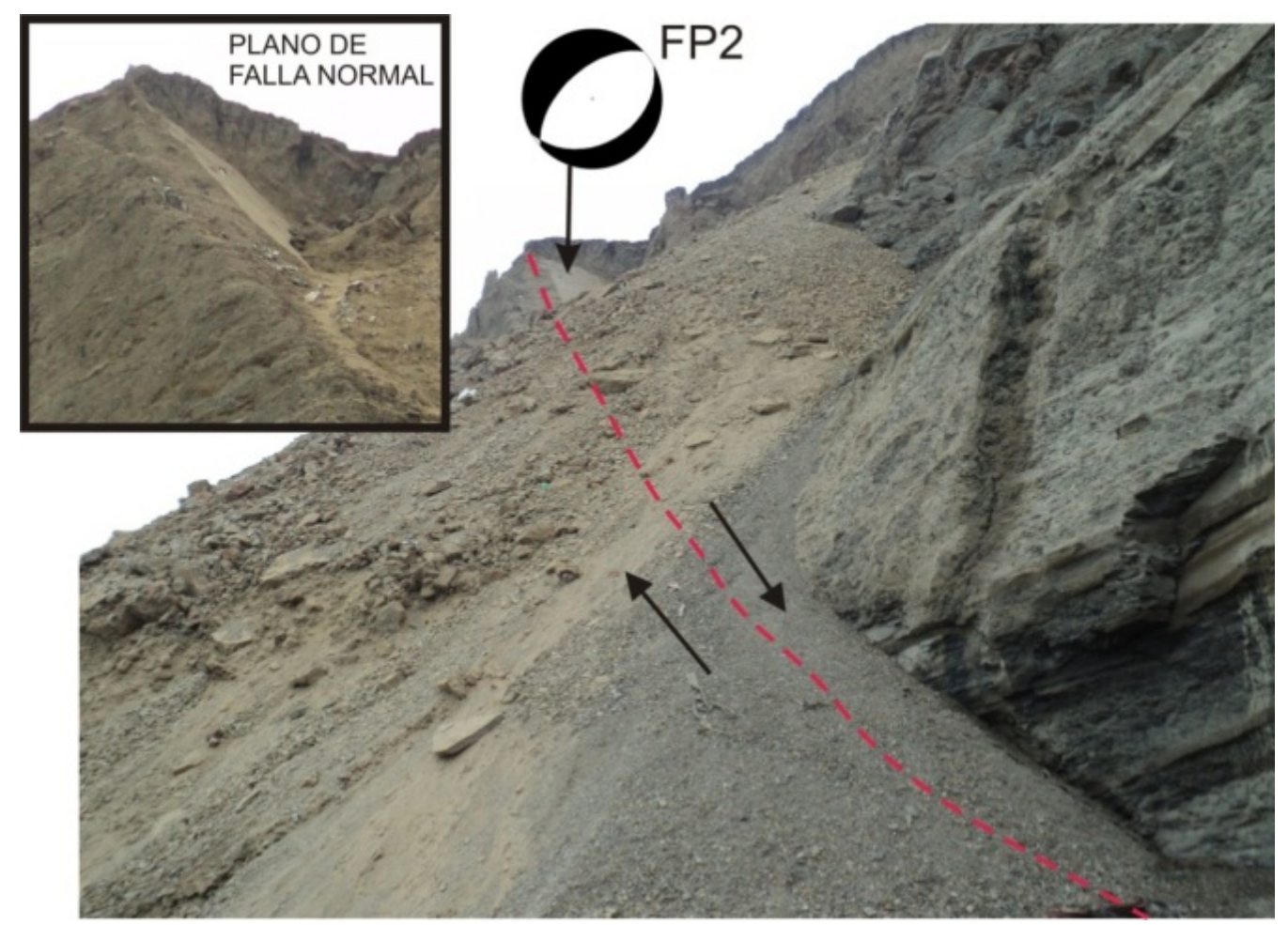

Figura 6. Evidencia de un plano de falla normal donde se desplazan lístricamente estratos rocosos.

\section{Deslizamiento del 07.04.2011}

Como indicado en la primera sección de este informe, el 07 de Abril de 2011, a las 06h50, unnuevo deslizamiento aconteció en el sector "2 de Febrero", la dimensión dedeslizamiento es mayor que el ocurrido en mayo 2010 y el 12 de Agosto de 2010. En la Figura 7 sepuede estimar la dimensión del material desprendido y su asentamiento al pie deacantilado.

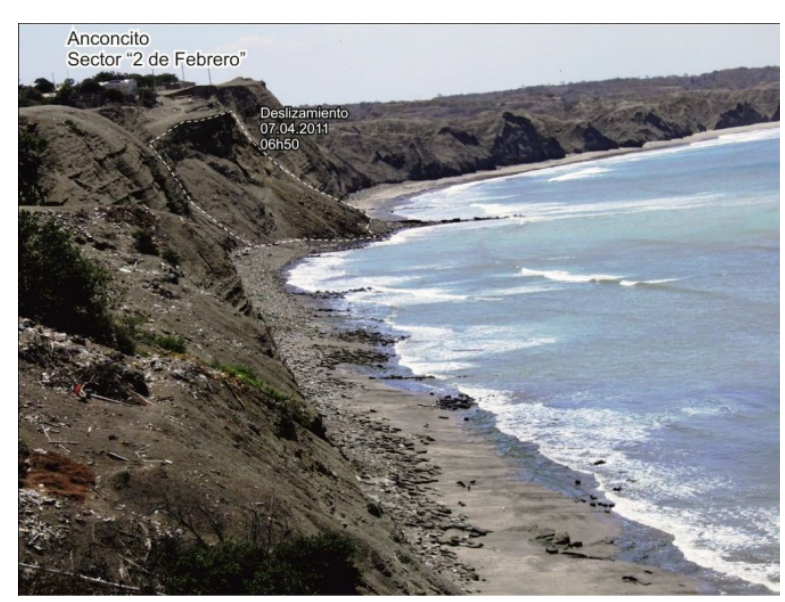

Figura 7. Se puede observar la dimensión del deslizamiento hacia el pie del acantilado,

en superficie es notable viviendas habitables que están dentro del área de influencia directa.

En la Figura 8, se puede observar el cambio brusco de la superficie del terreno, lasdimensiones de la tasa de desplazamientos aún están en movimiento hasta alcanzar losniveles de “asentamiento", las grietas visiblemente alcanzan los 10 metros deprofundidad, sin embargo su tendencia estructural se acerca a la base del acantilado.

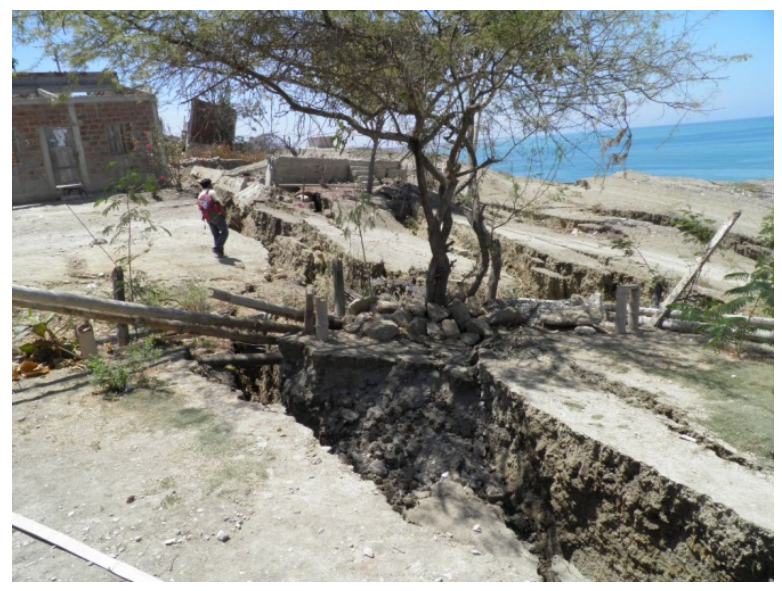

Figura 8. Grietas profundas alcanzan el metro de ancho y cerca de 10 metros deprofundidad, producto del desprendimiento del 07.04.2011.

La delimitación del alcance de fractura o grieta no indica el límite más estable, como loindicado en las bandas señaléticas en amarillo de "peligro", al contrario es urgenteestablecer medidas adecuadas y salvaguardar algunas familias que habitan en el áreacrítica (indicada como área de influencia directa de este fenómeno geológico y señaladatambién en el informe de la UPSE de Agosto 2010). 


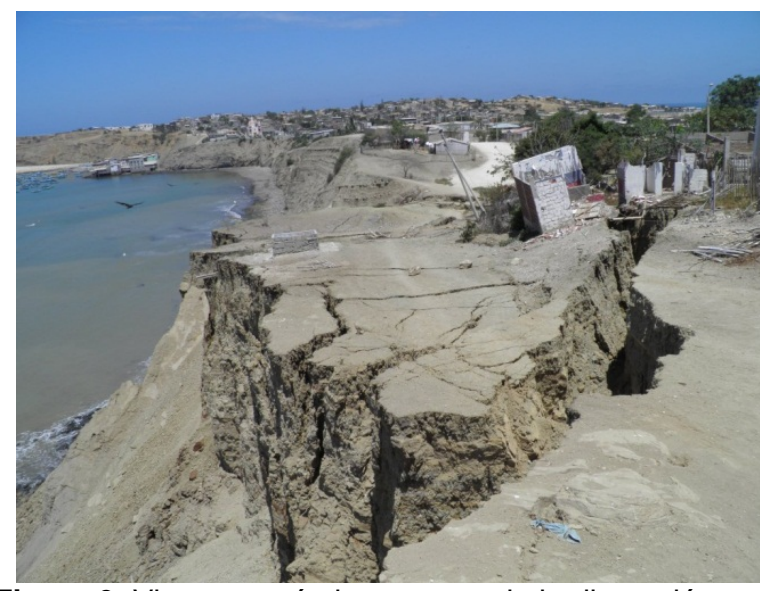

Figura 9. Vista panorámica mostrando la dimensión del desprendimiento del acantiladoy el colapso de estructuras edilicias.

Hasta ahora han sido mencionado las evidencias y deformaciones del terreno ensuperficie, sin embargo son presentado también en este artículo, las evidencias defracturas por tensión en la base del acantilado (Figura 5). Estas fracturas sonconsecuencia de la perdida de resistencia del talud que asociado a la alta tasa de erosiónmarina que socava las paredes provocan una rápida inestabilidad y posterior colapso.

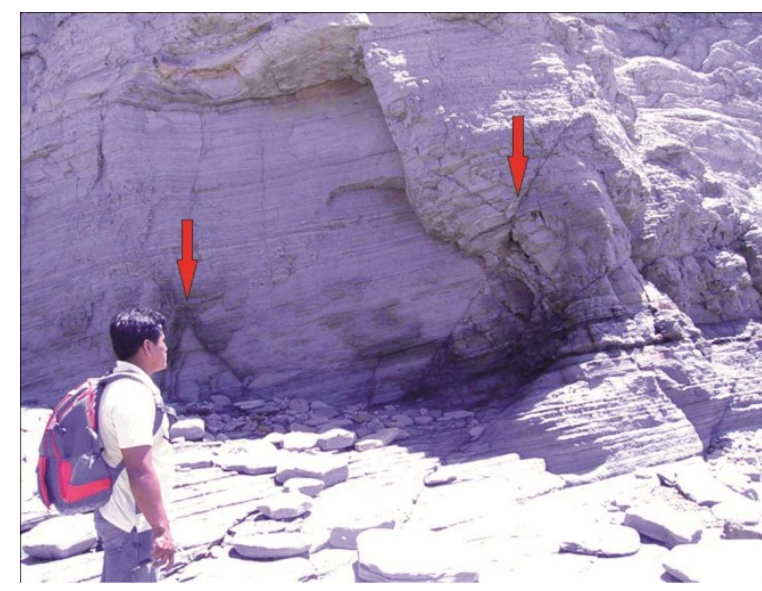

Figura 10. Fracturas por tensión en la base del acantilado.

La tendencia estructural del acantilado es de N235 y los estratos de las secuenciasrocosas buzan o se inclinan 290/10 (al NW), esta información nos indica que los estratostienen dirección contraria a los planos de deslizamiento; entonces el problema seincrementa con las fallas geológicas de tipo normal, paralelo a la dirección de la tasa demovimiento, que permiten la reactivación a lo largo de los planos de deslizamientos.



Figura 11.Material rocoso deslizado y desprendido en el sector "2 de Febrero".

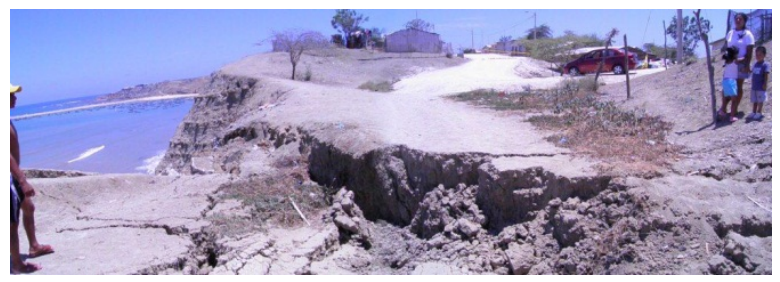

Figura 12. Vía o acceso vehicular construido en Agosto y Septiembre de 2010, la foto indica que la obra no tuvo planes de prevención y selección de sitios adecuadas para construcción vial.

\section{Conclusión}

Estudios geológicos que permitan delimitar sectores no apropiados para construcciones viales no han sido realizados para el sector "2 de Febrero". Fenómenos de desprendimientos y deslizamientos de estratos rocosos ya eran evidentes en mayo a agosto del 2010, en el mismo lapso de tiempo se iniciaban los trabajos viales con maquinarias pesadas sin considerar la carga estática y vibraciones transmitidas en el terreno.

La dinámica de erosión marina en los acantilados ha provocado socavamiento y pérdida de resistencia de los estratos más blandos, seguidos por desprendimientos de estratos rocosos y acumulación de material en la zona de playa.

Desde un punto de vista del análisis geológico estructural, el volumen total estimado que se ha deslizado el 07 de Abril de 2011 es de $61.175 \mathrm{~m}^{3}$; sinembargo recordemos que el área critica donde inter-actúan los tresplanos de fallas es mayor de aproximadamente $306.305 \mathrm{~m}^{3}$.

El comportamiento geomecánico del deslizamiento es complejo, por unaparte es de tipo rotacional y es también evidente los desprendimientos de bloques de losacantilados, se ha considerado la cinemática de las fallas geológicas yde las fracturas de tensión presentes en el área (conociendo lasdiferentes direcciones de esfuerzos de tracción), ya que son los principales 
planos dedeslizamientos que están deformando y colapsando el terreno.

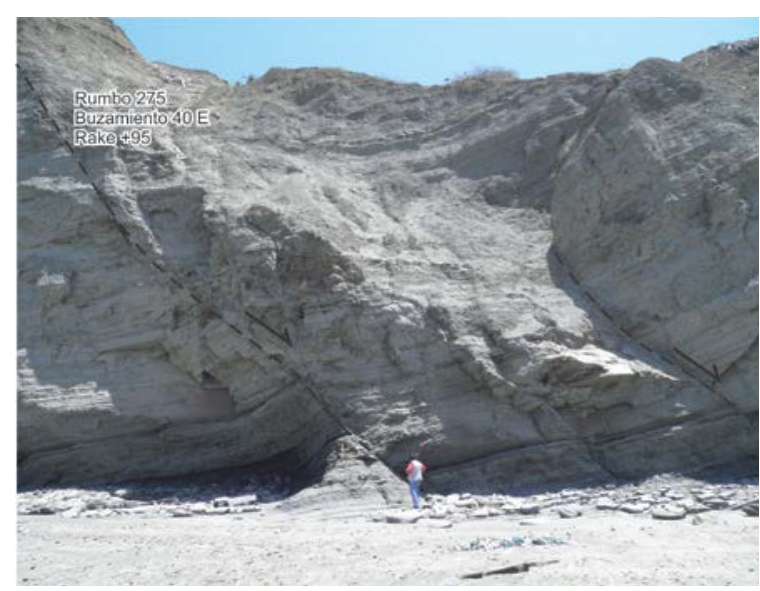

Figura 13.Fallamiento en el acantilado con desplazamientos transtensivos. Evidencia que indica una alta tasa de inestabilidad en el sitio "2 de Febrero".

Estos fenómenos geológicos son asociados a movimientos laterales transtensivos que se presentan previamente como grietas profundas evidenciadas en los escarpes de deslizamientos. Las velocidades de los desplazamientos pueden incrementarse por la infiltración durante altas precipitaciones en las estaciones de invierno, por moderados sismos locales $\mathrm{Mw} \approx 5$ o también por fuertes terremotos lejanos $7 \leq \mathrm{M} \leq 8.8$. M, magnitud del sismo.

Localmente, los daños son evidentes en la superficie donde estructuras físicas (ie. viviendas, instituciones educativas, etc.) de concreto son cortadas desde sus bases, afectando directamente a 35 familias asentadas cerca del borde de acantilado hasta el límite externo de la vía en construcción.

\section{Agradecimiento}

El Autor agrade el soporte logístico de la Facultad de Ciencias de la Ingeniería de la Universidad Estatal Península de Santa Elena, a través de sus Directivos Ingenieros Ramón Muñoz, Alamir Álvarez y Juan Garcés. Igual agradecimiento al Sr. Carlos Zambrano por el levantamiento topográfico.

\section{Referencia bibliográfica}

Baldock, J.W., 1982. Geología del Ecuador: Boletín de la explicación del Mapa Geológico de la República del Ecuador, Esc. 1:1,000,000. Min. Rec. Nat. Energ., Quito, 10., Quito.

Baldock, J. W. (1983), The Northern Andes: A review of the Ecuadorian Pacific Margin, in The Oceans Basins and Margins, edited by A. E. M. Nairn,
F. G. Stehli, and S. Uyeda, Plenum Press, New-York and London, 181- 271.

Bristow, C.R., Hoffstetter, R., 1977. Lexique Stratigraphique, Amérique Latine. Ed. CNRS, Paris, Fas. 5-2, Equateur: 410.

IAEA Safety Standards Series (2002).Evaluation of Seismic Hazards for Nuclear Power Plants.Safety Guide. No. NS-G-3.3. International AtomicEnergy Agency.

Núñez del Arco E., Dugas F., Labrousse B. (1983). Contribución al conocimiento estratigráfico, sedimentológico y tectónico de la región oriental de la Península de Santa Elena y Parte del Sur de la Cuenca del Guayas (Ecuador) en base a 17 hojas geol[ogicas escala 1/50.000. Actas del III Congreso Ecuatoriano de Ingeniería, Geología, Minería y Petróleo, Guayaquil, v. IB., 33p.

Robert, G.P., Michetti, A.M. (2004). Spatial and temporal variations in growth rates along active normal fault systems: an example from The Lazio Abruzzo Apennines, central Italy. Journal of Structural Geology 26, 339-376.

Sheppard, George. (1946). The geology of the Guayaquil estuary, Ecuador. Journal of the Institute of Petroleum, Institute of Petroleum, London, United Kingdom (GBR), Vol.32, no.272, pp.492-514.

SlemmonsD.Burton and Depolo Craig M. (1986). Evaluation of active faulting and associated hazards.Active Tectonics: ImpactonSociety, 45-62. 